\title{
Demonstration of the Space Launch System Augmenting Adaptive Control Algorithm on Pole-Cart Platform
}

\author{
Jing Pei* Paul Rothhaar ${ }^{\dagger}$ \\ NASA Langley Research Center, Hampton, Virginia, 23681
}

\begin{abstract}
NASA's baseline Space Launch System (SLS) flight control system (FCS) includes an adaptive augmenting control (AAC) portion in addition to the flight-heritage nominal classical controller. The AAC algorithm is intended to improve the robustness and performance of the classical controller. Over the past several years, the AAC algorithm developed at NASA Marshall Space Flight Center (MSFC) has matured significantly through extensive simulation, rigorous analytical proofs, and a series of successful flight tests on a F18 aircraft. This study was part of a SLS program and NASA Engineering and Safety Center (NESC) joint effort to further increase the confidence level of the AAC algorithm by demonstrating its key functionalities on a classroom type of example, the pole-cart system, at the NASA Langley Research Center (LaRC) dynamics and control laboratory. The fundamental dynamics behind balancing an inverted pendulum is similar to controlling an aerodynamically unstable rocket. Both systems are inherently open-loop unstable and requires feedback control for attitude stabilization. The principles behind the AAC algorithm is applicable to a wide range of conditionally stable dynamical systems. Hence, the outcomes from this simple and inexpensive exercise has provided the SLS program with additional confidence into the AAC design, operation, robustness, and application.
\end{abstract}

\section{Introduction}

NASA's Space Launch System (SLS) Flight Control System (FCS) architecture is mainly based on a classical gain-scheduled control design with extensive flight heritage. Recently, the SLS program has baselined an Adaptive Augmenting Control (AAC) algorithm that is intended to enhance the robustness of the classical controller without adversely impacting performance when the vehicle is operating within its design envelope. ${ }^{1,2}$ Over the past several years, the AAC algorithm has matured significantly through extensive simulation, rigorous analytical proofs, and a series of successful flight tests on a F/A-18 aircraft. ${ }^{3,4}$ The fundamental principles behind the AAC algorithm suggest that it should be applicable to a wide range of conditionally stable dynamical systems. The inverted pendulum (IP) on a cart (pole-cart) is a classical system widely studied in academia ${ }^{5-8}$ and control theory. Similar to an aerodynamically unstable rocket, the inverted pendulum (IP) is open-loop unstable and requires active feedback for stabilization. This study was part of a SLS program and NASA Engineering and Safety Center (NESC) joint effort to further increase the confidence level of the AAC algorithm by demonstrating its key functionalities on a classroom type of example, the pole-cart system, at NASA Langley Research Center (LaRC) dynamics and control laboratory.

This paper describes how the pole-cart hardware was used to demonstrate the three primary objectives of the SLS AAC algorithm. ${ }^{1-3,9,10}$ These objectives are: 1) "do no harm", minimal adaptation when the baseline classical controller is performing nominally; 2) Increase system gain in response to excessive command tracking errors in the presence of mismodeled dynamics or in-flight anomalies; 3) Decrease system gain to prevent undesireable high frequency parasitic dynamics (i.e control structure interaction) from driving the system to instability. The remainder of this paper is organized as follows: Section II derives the equations of motion (nonlinear and linear) for the pole-cart system and provides an analogy between itself and an aerodynamically unstable rocket. Section IV shows the nominal stability margins of the system. Section III

\footnotetext{
*Aerospace Engineer, Systems Analysis and Concepts Directorate, Vehichle Analysis Branch

${ }^{\dagger}$ Aerospace Engineer, Research Directorate, Dynamics and Control Branch
} 
discusses the experimental setup and system parameters. Section V describes how the SLS AAC parameters were re-adjusted for the pole-cart dynamics. Results from the experiment and numerical simulations are shown. Section VI summarizes the results and provides discussion for potential future work.

\section{Pole-Cart Dynamics}

The pole-cart system is a classical setup used in academia to illustrate principles of controls engineering. The objective is to move the cart back and forth on the track to actively stabilize the inverted pendulum (IP). The IP is an inherently unstable system and can be used to represent the planar dyamics of a typical aerodynamically unstable launch vehicle. Both systems have a real pole on the right half of the s-plane and require active feedback control for stabilization. The cart is analogous to the control effector(s) that are located at the base of a launch vehicle. Figure 1 is an illustration showing the similarities between the two systems.

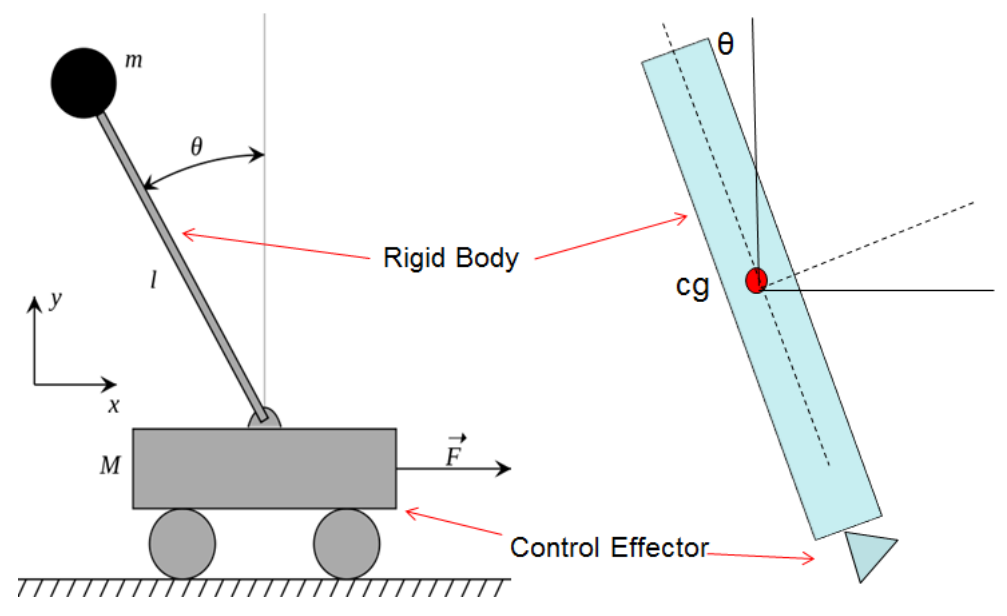

Figure 1. Dynamic Similarity Between Pole-Cart and Launch Vehicle

Lagrange's method ${ }^{11}$ was used to derive the equations of motion (EOM) of the cart and pendulum configuration. Figure 2 shows the nomenclature and sign conventions that were adopted in the derivation. $m_{c}, m_{p}$ are the mass of the cart and IP respectively. $l_{p}$ is the total length of the pendulum. $J_{p}$ is the inertia of the pendulum about the center of mass. Equations 1 through 6 illustrates the nonlinear system of equations and follow the assumption that the mass is distributed uniformly so that the mass center is located at $l_{p} / 2$ from $\mathrm{P}$.

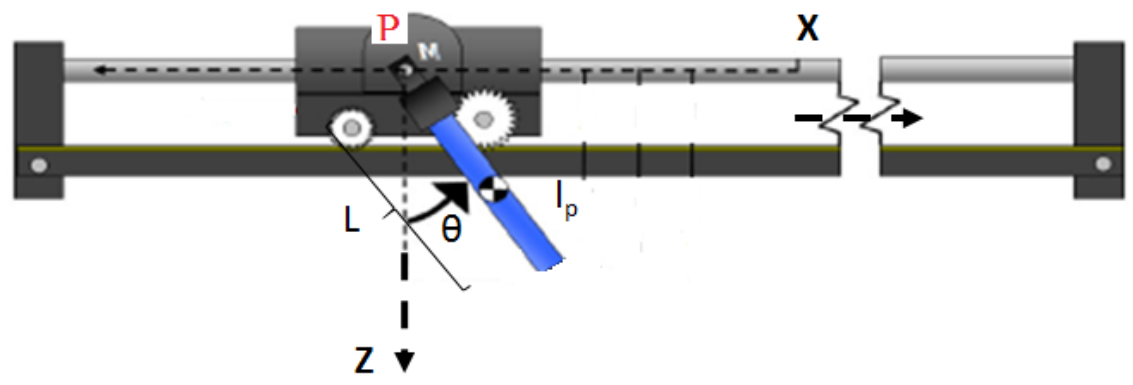

Figure 2. Nomenclature and Sign Conventions

Using the angular orientation, axes, and lengths from Fig. 2, the Lagrangian can be expressed as the total kinetic $(T)$ and potential $(V)$ energy of the system shown in Eq. (2) and the generalized coordinates of the system are: $x$ and $\theta$. 


$$
\begin{aligned}
L=T-V & =\frac{1}{2}\left(m_{p}+m_{c}\right) \dot{x}^{2}+\frac{1}{8} m_{p} l_{1}^{2} \dot{\theta}^{2}+\frac{1}{2} m_{p} l_{p} \dot{x} \dot{\theta} \cos \theta \\
& +\frac{1}{2} J_{p} \dot{\theta}^{2}+\frac{1}{2} m_{p} g l_{p} \cos \theta
\end{aligned}
$$

Following Lagrange's approach, the final nonlinear equations of motion can be shown in a matrix representation shown in Eq. (2). The states are the generalized coordinates and their time derivatives.

$$
\left[\begin{array}{l}
\ddot{x} \\
\ddot{\theta}
\end{array}\right]=\left[\begin{array}{ll}
a_{11} & a_{12} \\
a_{21} & a_{22}
\end{array}\right]^{-1}\left[\begin{array}{l}
b_{1} \\
b_{2}
\end{array}\right]
$$

where $\mathrm{A}$ is the matrix with elements aij which are:

$$
\begin{aligned}
& a_{11}=m_{p}+m_{c} \\
& a_{12}=\frac{1}{2} m_{p} l_{p} \cos \theta \\
& a_{21}=\frac{1}{2} m_{p} l_{p} \cos \theta \\
& a_{22}=\frac{1}{4} m_{p} l_{p}^{2}+J_{p}
\end{aligned}
$$

and the terms $b_{1}, b_{2}$ are:

$$
\begin{aligned}
& b_{1}=F+\frac{1}{2} m_{p} l_{p} \sin \theta \dot{\theta}^{2} \\
& b_{2}=\tau-\frac{1}{2} m_{p} l_{p} g \sin \theta
\end{aligned}
$$

The forces and torques include both the motor force $\left(f_{\text {motor }}\right)$ and system friction coefficients $\left(k_{\dot{x}} \dot{x}, k_{\dot{\theta}}\right)$ :

$$
\begin{aligned}
F & =k_{\dot{x}} \dot{x}+f_{\text {motor }} \\
\tau & =k_{\dot{\theta}} \dot{\theta}
\end{aligned}
$$

The inverse of the non-linear state dependent mass matrix comprised of elements $a_{i j}$ is computed at each integration interval for simulation. In order to mimic the short period dynamics of a launch vehicle, the nonlinear cart pendulum EOM were linearized about the desired operating condition: $x=0, \theta=\pi, \dot{x}=0$, $\dot{\theta}=0$ and put into the standard state space $\dot{x}=A x+B u$ format. Equations 7 through 10 are elements of the linearized $\mathrm{A}$ and $\mathrm{B}$ matrices without motor dynamics.

$$
A=\left[\begin{array}{cc}
0_{2 \times 2} & I_{2 \times 2} \\
-H^{-1} \frac{\partial G}{\partial q} & 0_{2 \times 2}
\end{array}\right]
$$

where $\mathrm{H}$ is the inverse of the linearized A matrix and $\frac{\partial G}{\partial q}$ are the stiffness terms dependent on $\theta$

$$
\left.\begin{array}{c}
H_{11}=m_{p}+m_{c} \\
H_{12}=-\frac{1}{2} m_{p} l_{p} \\
H_{21}=-\frac{1}{2} m_{p} l_{p} \\
H_{22}=\frac{1}{4} m_{p} l_{p}^{2}+J_{p} \\
\frac{\partial G}{\partial q}=\left[\begin{array}{cc}
0 & 0 \\
0 & -\frac{1}{2} m_{p} l_{p} g
\end{array}\right] \\
B=\left[\begin{array}{cc}
{\left[\begin{array}{c}
0_{2 \times 1} \\
H^{-1}
\end{array}\right]} \\
1 \\
0
\end{array}\right]
\end{array}\right]
$$


The motor dynamics can be approximated by Eq. (11) in series with a second-order transfer function, where $F$ is the input force to the cart, $V$ is the commanded voltage, $K_{m}$ is the back EMF constant, $K_{g}$ is the gearbox ratio, $R_{m}$ is the motor armature resistance, and $r$ is the wheel radius. The second-order transfer function has a natural frequency, $\omega_{n}$, of $54 \mathrm{rad} / \mathrm{s}$ and a damping ratio, $\zeta$ of 0.5 . These parameters were determined through a series of system identification experiments.

$$
F=\frac{K_{m} K_{g}}{R_{m} r} V-\frac{1}{R_{m}}\left(\frac{K_{m} K_{g}}{r}\right)^{2} \dot{x}
$$

\section{Experimental Setup}

Figure 3 shows the pole-cart system setup at the LaRC Dynamic Sytems and Control Branch's (DSCB) Aircraft Controls Research Laboratory (ACRL). The setup consists of a host computer, a target computer, a data acquisition board, input/output connection block, power unit that drives the motor, and the cart pendulum hardware. The cart position and the angle of the inverted pendulum are sensed using two quadrature optical encoders. The host computer is used for the design/modeling of the plant and controller. The target computer runs Mathworks XPC Target, which executes the model and controls the hardware in real-time.

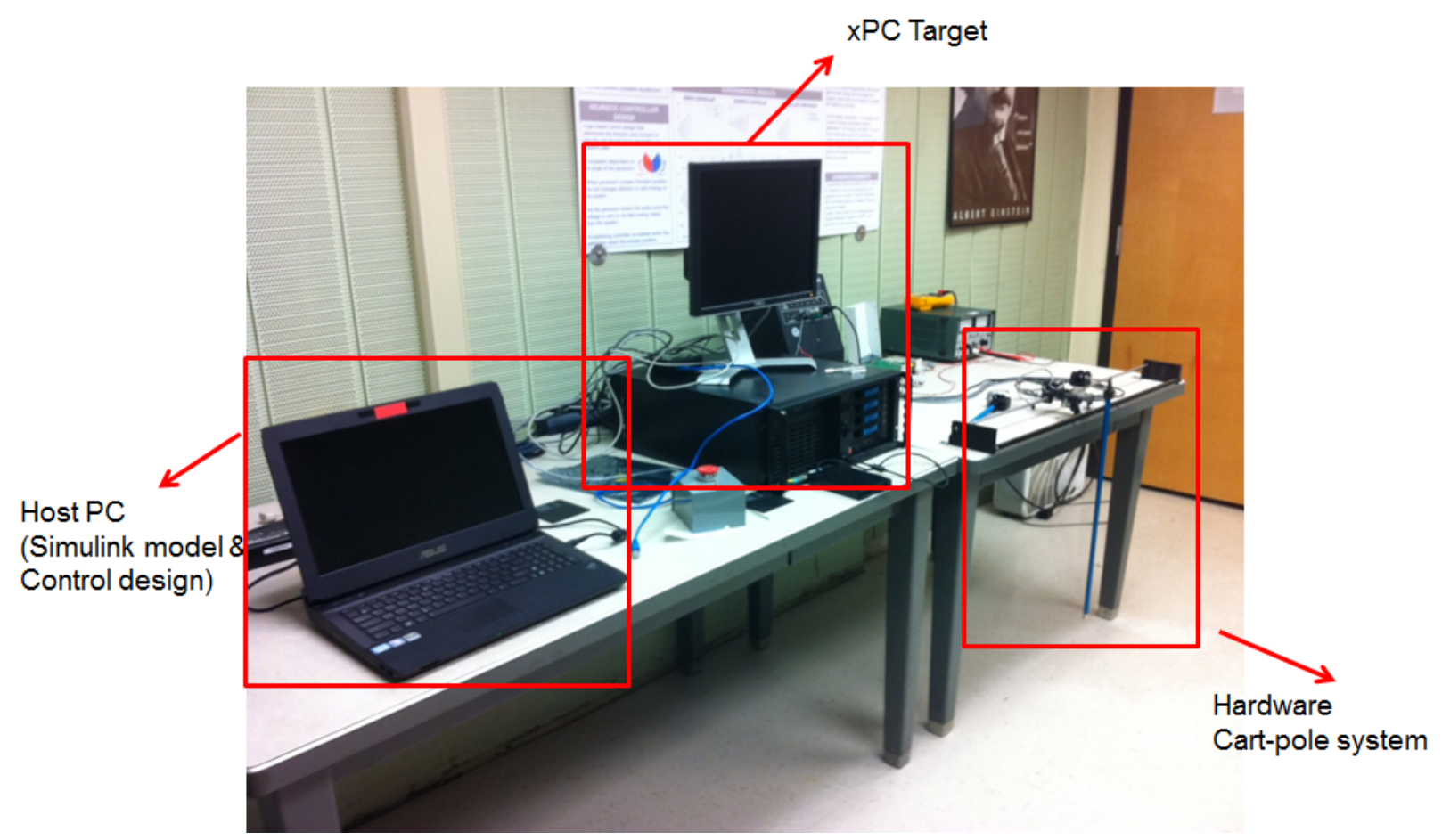

Figure 3. Experimental Setup

Constant parameters include mass of the cart, mass and length of the IP. Table 1 tabulates the value of each parameter. 
Table 1. System Parameters

\begin{tabular}{cc}
\hline \hline System Parameters & Values \\
\hline Mass Cart & $0.56 \mathrm{~kg}$ \\
Mass Inverted Pendulum & $0.28 \mathrm{~kg}$ \\
Length Inverted Pendulum & $0.64 \mathrm{~m}$ \\
Motor armature resistance, $R_{m}$ & $2.6 \Omega$ \\
Back EMF constant, $\mathrm{Km}$ & $0.00767 \mathrm{Nm} / \mathrm{A}$ \\
Gearbox ratio, $\mathrm{Kg}$ & 3.7 \\
Wheel Radius, $\mathrm{r}$ & $0.0064 \mathrm{~m}$ \\
\hline \hline
\end{tabular}

\section{Nominal Stability Margins}

Figure 4 is a block diagram of the system showing the nominal controller with the AAC portion circled in red. The "gain shift" block was used to adjust the nominal loop gain up or down intentionally to demonstrate the system performance with degraded low or high frequency stability margins. In the meanwhile, the AAC algorithm adjusts $K_{T}$ (a multiplicative gain adjustment) in real time to bring the system back within its nominal performance envelop.

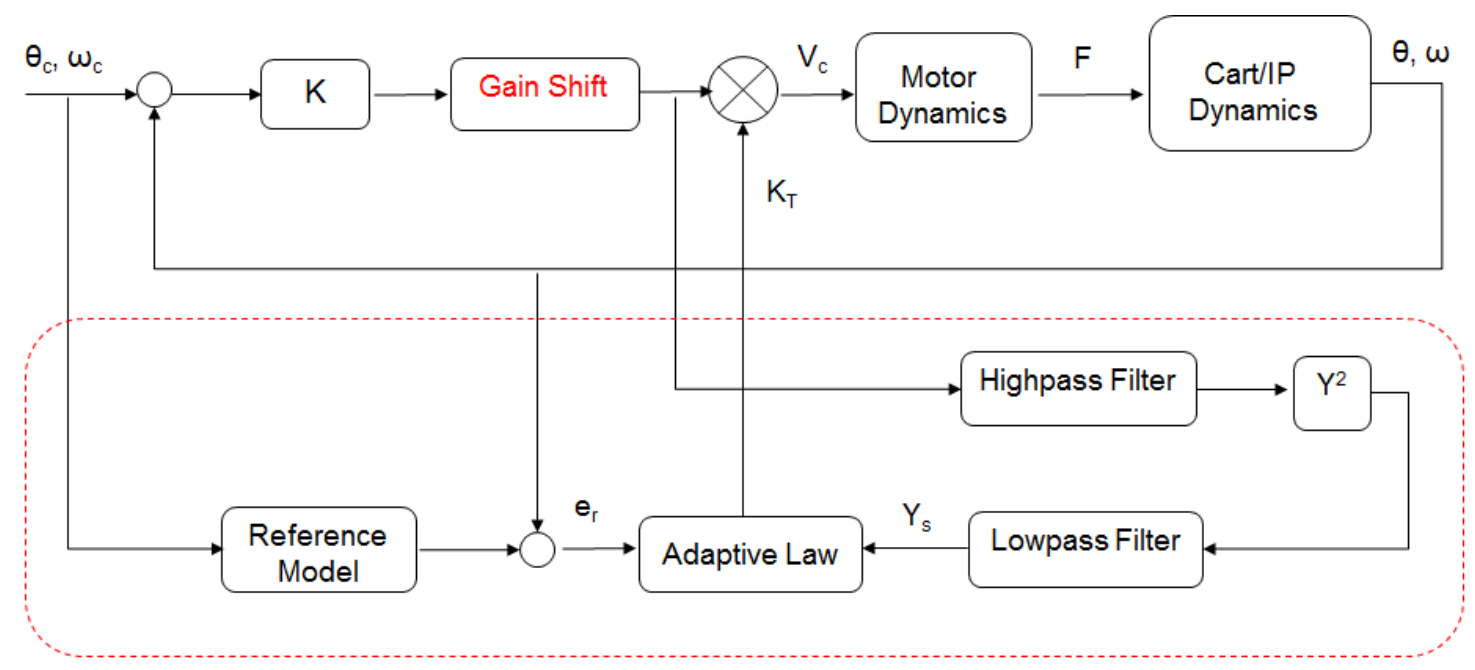

Adaptive Augmenting Control

Figure 4. Block Diagram of Pole-Cart System with AAC and Gain Adjustment Block

A Linear Quadratic Regulator (LQR) controller was designed to simultaneously stabilize the IP while keeping the cart at the center of the track. The cart postion/velocity $(x, \dot{x})$ and IP angle/angular velocity $(\theta, \dot{\theta})$ were available for feedback. The nominal feedback gains were: [-14.1 $48.4-16.8$ 8.4]. Figure 5 shows a root locus plot of the linearized system with varying forward loop gain or "gain shift" parameter. The shape of the root locus plot is remarkably similar to that of an aerodynamically unstable launch vehicle (in the absence parastic dynamics such as tail-wags-dog, slosh, and structural dynamics). The double integrator is associated with the cart dynamics. The IP dynamics are unstable (two real axis poles with one being on the positive real axis). The actuator dynamics start out as a pair of complex roots on the left-hand plane and migrate towards the imaginary axis with increasing foward loop gain. The closed-loop system is conditionally stable as long as the forward loop gain ("gain shift" parameter) remains within a certain range. 


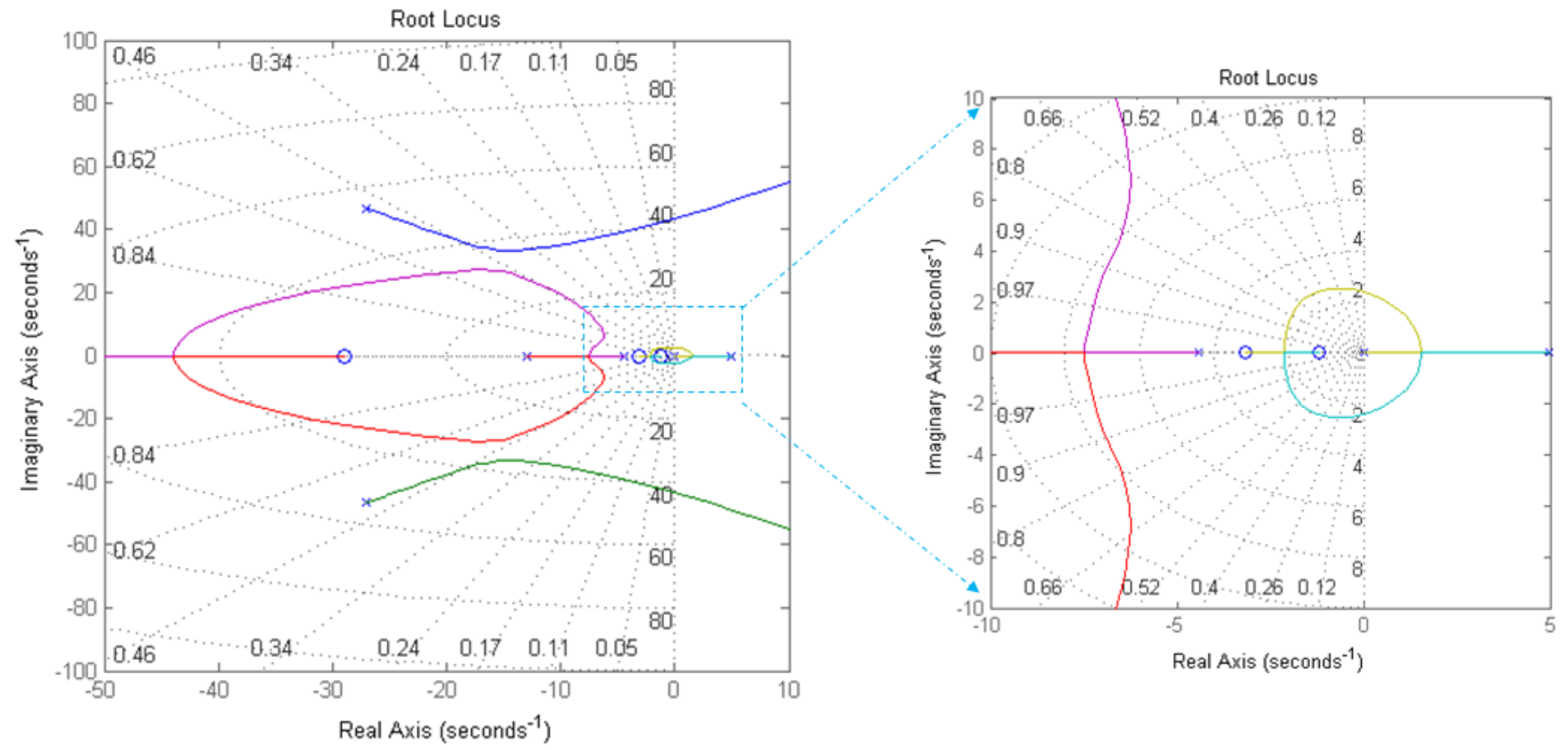

Figure 5. Root Locus of the Linearized Pole-cart system

Figure 6 shows the Nichols plot of the system with the standard $6 \mathrm{~dB}$ and $30 \mathrm{deg}$ gain and phase margin guidelines represented by the red circle. The analytical model suggests $-7.13 \mathrm{~dB}(\mathrm{x} 0.44)$ and $14 \mathrm{~dB}(\mathrm{x} 5)$ for the low frequency and high frequency gain margins, respectively. The gain crossover frequency occurs at $1.85 \mathrm{~Hz}$ with a phase margin of 35 degrees. For a typical aerodynamically unstable booster vehicle, the ratio of the gain crossover frequency to the unstable pole frequency is about $2: 1$. That ratio is $2.3: 1$ for the pole-cart system.

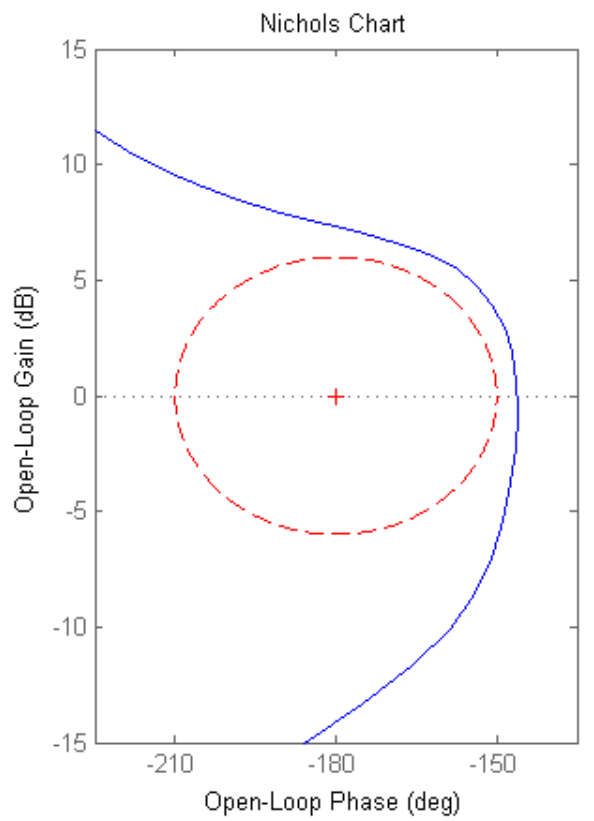

Figure 6. Nichols Chart of the Linearized Pole-cart system 
Figure 7 shows sample system time history from an experimental run with only the nominal baseline controller activated ("gain shift" parameter $=1$ ). Subplot 7.1 .1 is a phase portrait of pendulum states, $\dot{\theta}$ vs. $\theta$. Subplot 7.1 .2 is a phase portrait of cart states, $\dot{x}$ vs. $x$. The regulator did a good job of keeping the IP inverted at its unstable equilibrium point, $\pi$, and the cart in the middle of the track throughout the experiment. The input voltage stayed well below the \pm 4 volt saturation limit. Subplot 7.2 .2 shows the AAC-modulated loop gain, $K_{T}$ (remained at 1 since AAC was turned off in this example).
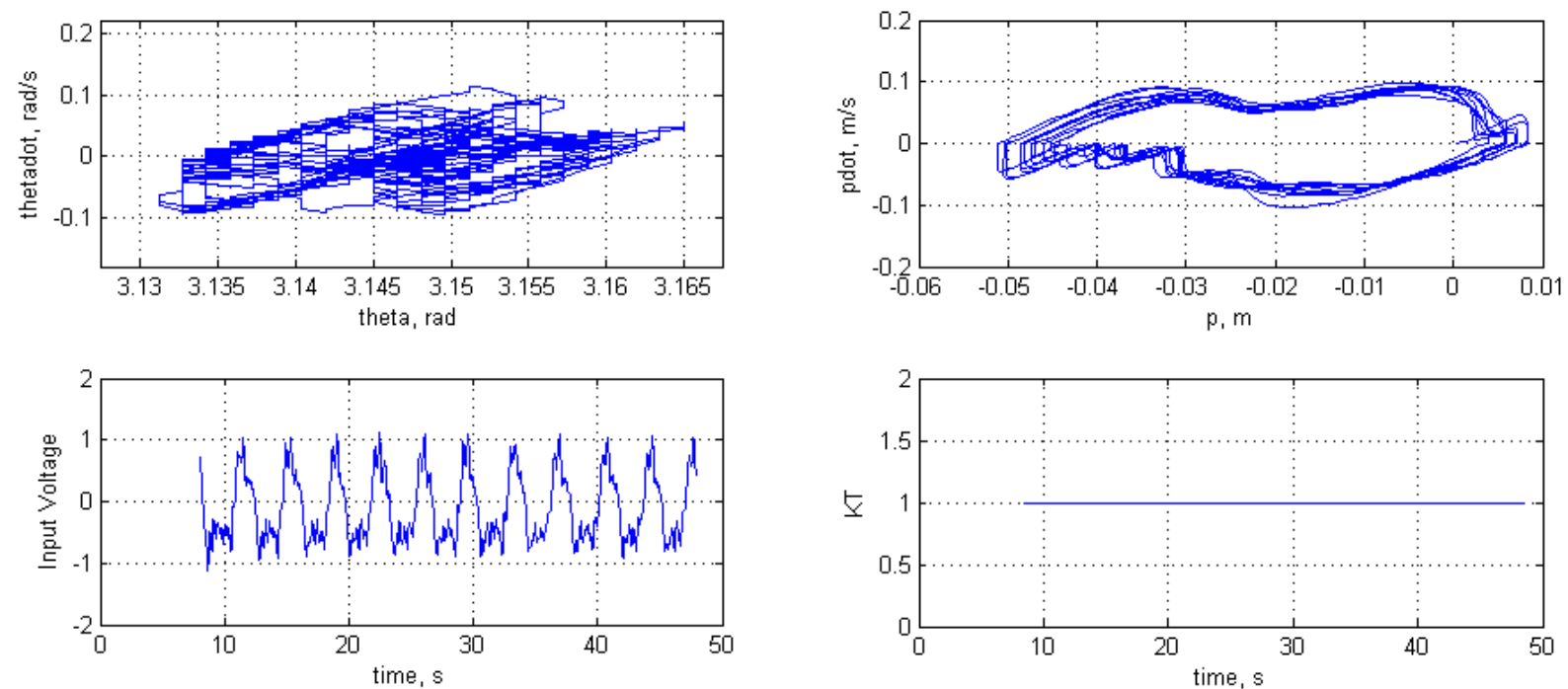

Figure 7. Hardware Nominal AAC OFF, "gain shift" parameter $=1(0 \mathrm{~dB})$

Tests (trial and error) were performed on the actual hardware to quantify the stability margins. The low frequency gain margin was determined to be $-5.7 \mathrm{~dB}(\times 0.52)$. This was found by gradually lowering the nominal forward loop gain or "gain shift" parameter, from the nominal value of 1 to 0.52 at which the onset of instability was observed. At this point, the cart was barely able to keep the IP upright. Figure 8 shows slow divergent oscillatory behavior of the IP angle and the cart position as the "gain shift" parameter was reduced by $5.7 \mathrm{~dB}$. The frequency of this instability is approximately $2.5 \mathrm{rad} / \mathrm{s}$, which is consistent with the frequency at which the pendulum poles cross the imaginary axis on the root locus plot shown in Figure 5 . The voltage input reached the \pm 4 volt saturation limit at around 25 seconds. 

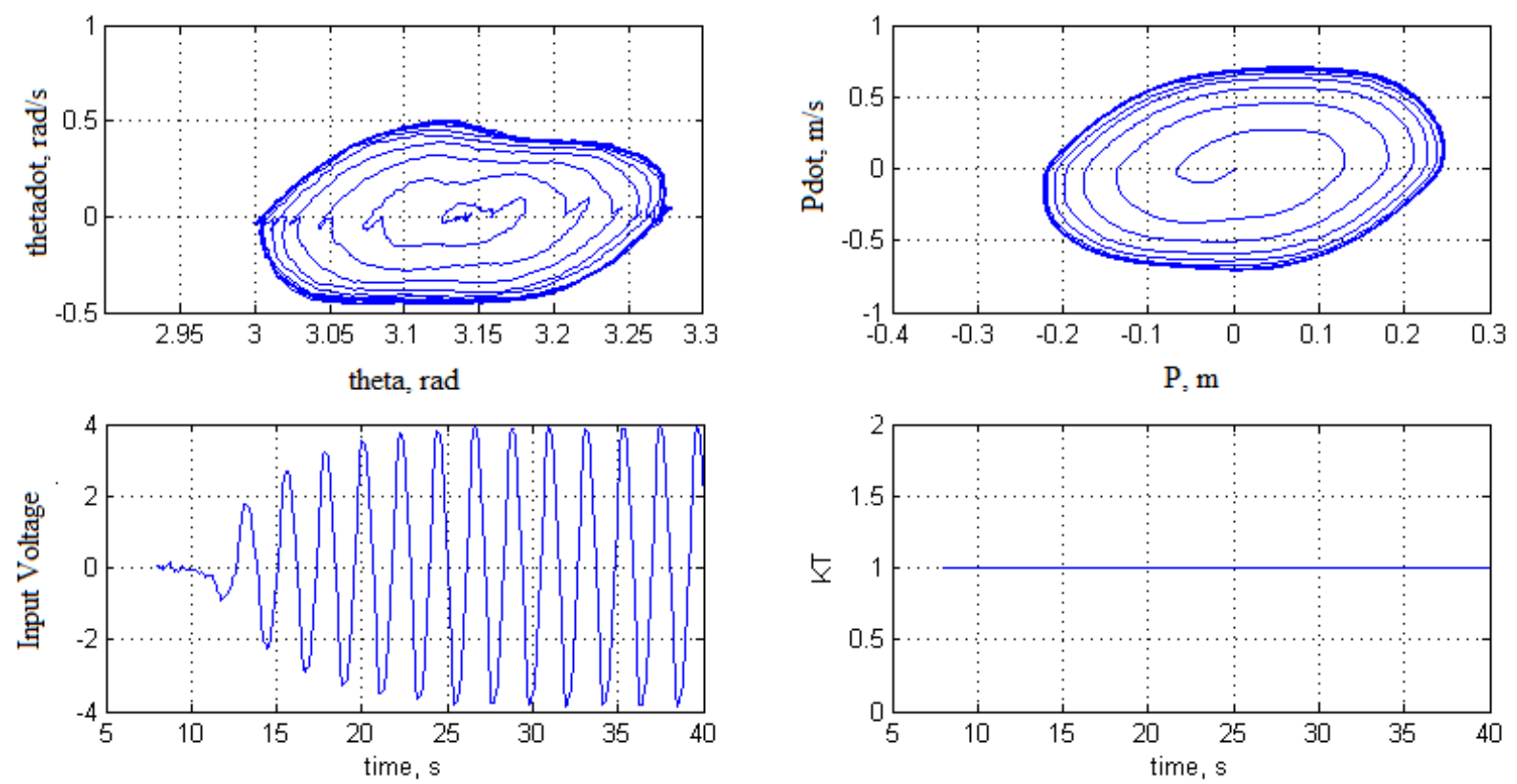

Figure 8. Hardware Low Frequency Instability, "gain shift" parameter $=0.52(-5.7 \mathrm{~dB})$

The hardware high frequency gain margin was determined to be $12.5 \mathrm{~dB}(\times 4.2)$. Figure 9 shows the behavior of the system when the high frequency instability was reached. The control input immediately reached the \pm 4 volt saturation limit and forced the pendulum and cart states into a persistent limit cycle oscillation (LCO). Power spectral density analysis of the data showed the frequency of oscillation occuring approximately at $7 \mathrm{~Hz}$. This is consistent with the simulated high frequency gain margin and the crossing of the imaginary axis in the root locus plot at just above $40 \mathrm{rad} / \mathrm{sec}$ shown by the blue and green loci in Figure 5 .
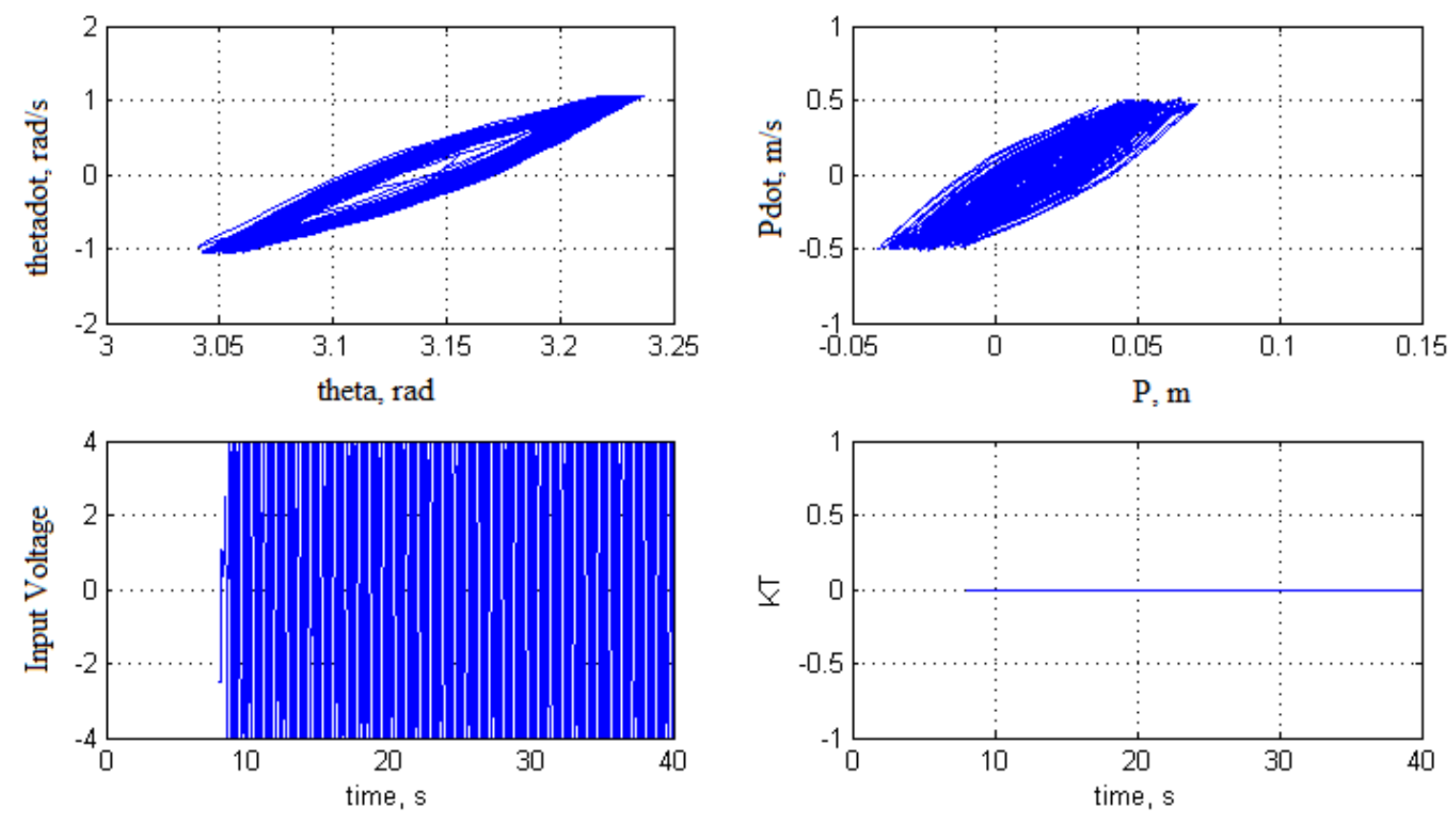

Figure 9. Hardware High Frequency Instability, "gain shift" parameter $=4.2(12.5 \mathrm{~dB})$ 
There appeared to be a $15 \%$ discrepancy between the stability margins predicted by the simulation model vs. actual hardware. These differences were most likely due to unmodeled dynamics and are shown in Table 2.

Table 2. Discrepancy between Simulation and Hardware

\begin{tabular}{ccccc}
\hline \hline Models & LF GM & HF GM & HF GM Freq & Gain Crossover Freq \\
\hline Linear Model & 0.44 & 5 & $7 \mathrm{~Hz}$ & $1.85 \mathrm{~Hz}$ \\
Nonlinear Simulation & 0.44 & 5 & $7 \mathrm{~Hz}$ & $1.85 \mathrm{~Hz}$ \\
Hardware & 0.52 & 4.2 & $7 \mathrm{~Hz}$ & $-\mathrm{Hz}$ \\
\hline \hline
\end{tabular}

\section{Results}

Readers should refer to Refs. 1,2,9,10 for the in-depth mathematics, analysis, and discussions behind the AAC algorithm. Since the pole-cart dynamics are roughly an order of magnitude faster compare to that of SLS (i.e., the gain crossover frequency is approximately $0.2 \mathrm{~Hz}$ for SLS and $1.85 \mathrm{~Hz}$ for pole-cart), most of the pole-cart AAC gains and filters were initially scaled by a factor of 10 (from the SLS design) and tuned manually based on observations made during the experiments. Table 3 compares AAC parameters used for SLS with those used for the pole-cart experiment. The adaptive law is a balance between low frequency tracking errors (reference model component) with the amount of high frequency contents in the control signal due to undesirable parasitic dynamics (spectral damper component). The analysis in Ref. 9 discusses the frequency domain behavior of the AAC and the concept of a frequency threshold or "selectivity zero". An overall error occurring lower in frequency than the "selectivity zero" will cause the AAC to increase the forward loop gain $\left(K_{T}>1\right)$, whereas an overall error above the "selectivity zero" frequency will cause the AAC to decrease the forward loop gain $\left(K_{T}<1\right)$. If the baseline controller is performing within the nominal envelope minimal adaptaion is expected $\left(K_{T} \approx 1\right)$. The adaptation limit of the $\mathrm{AAC}$ is $\pm 6 \mathrm{~dB}(0.5$ to 2$)$ which is based on the classical stability margin requirements.

Table 3. AAC parameters

\begin{tabular}{ccc}
\hline \hline Parameters & SLS & Pole-cart \\
\hline Gain crossover frequency, $\mathrm{Hz}$ & 0.2 & 1.85 \\
Spectral damper high pass filter $\omega_{n}, \mathrm{~Hz}$ & 0.4 & 6.8 \\
Spectral damper high pass filter $\zeta$ & 0.9 & 0.9 \\
Spectral damper low pass filter $\omega_{n}, \mathrm{~Hz}$ & 0.25 & 2.5 \\
Spectral damper low pass filter $\zeta$ & 1 & 1 \\
Error low pass filter $\omega_{n}, \mathrm{~Hz}$ & 0.2 & 2 \\
Error low pass filter $\zeta$ & 1 & 1 \\
K, ratio between Err and SD gain & 1.45 & 0.17 \\
a, Err gain & 100 & 10 \\
Selectivity function zero, Hz & 0.35 & 6.8 \\
AAC low pass filter $\omega_{n}, \mathrm{~Hz}$ & 0.15 & 1.25 \\
AAC low pass filter $\zeta$ & 1 & 1 \\
\hline \hline
\end{tabular}

Initially, the associated "selectivity zero" was placed slightly above the gain crossover frequency at 2.2 Hz. During hardware testing, it was discovered that a slight increase in the "gain shift" parameter led to a substantial drop in the AAC-augmented $K_{T}$, which was not encountered during simulations or previous studies. A Fast Fourier Transform (FFT) was performed on the control signal and subsequently indicated unknown source of noise that is between the "selectivity zero" frequency $(2.2 \mathrm{~Hz})$ and the high frequency gain margin $(7 \mathrm{~Hz})$. A slight increase in the "gain shift" parameter caused an amplification of the unknown noise to come back through the control path and subsequently caused an undesirable drop in the AAC-augmented $K_{T}$. A quick (ad-hoc) solution to the problem was to shift the "selectivity zero" to be slightly below the high 
frequency gain margin frequency at $6.8 \mathrm{~Hz}$. In essence, this forced the AAC to reduce $K_{T}$ only when the known actuator instability $(7 \mathrm{~Hz})$ is about to be reached. Figure 10 is a Nichols plot showing the updated location of the "selectivity zero".

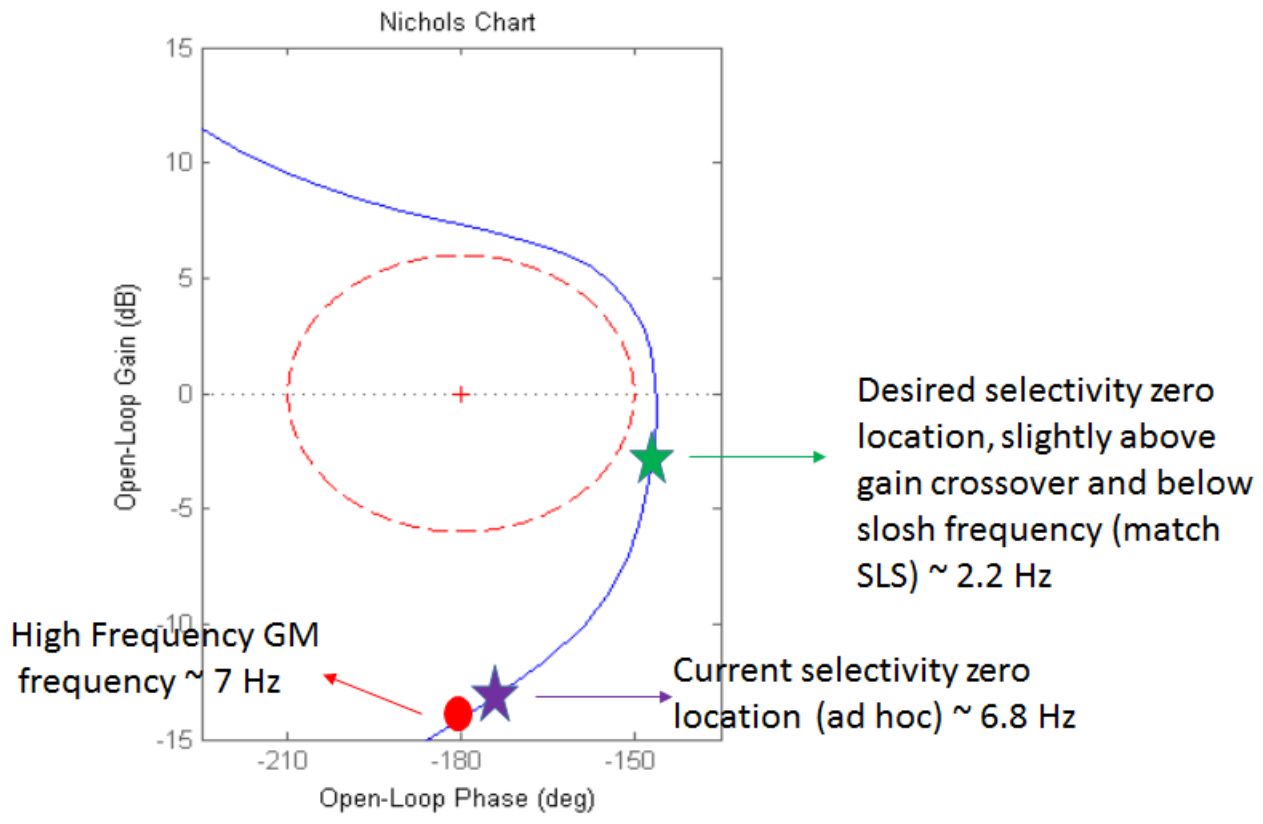

Figure 10. "Selectivity" Zero Location

The three key features of the AAC algorithm were demonstrated both experimentally and in simulation on the pole-cart dynamics: 1) "do no harm", minimal adaptation when the baseline classical controller is performing nominally $\left(K_{T} \approx 1\right)$. In this case, "gain shift" parameter was set to 1.2 ) Increase system gain in response to excessive command tracking errors in the presence of mismodeled dynamics or in-flight anomalies. In this case, the performance of the nominal controller was degraded such that it was no longer able to stabilize the system ("gain shift" parameter $<0.52$ ). The AAC was expected to increase $K_{T}$ such that the system regains stability $\left(K_{T}>1\right)$. 3) Decrease system gain to prevent undesireable high frequency parasitic dynamics (i.e control structure interaction) from driving the system to instability. In this case, the "gain shift" parameter was set to a value beyond the $\mathrm{x} 4.2$ high frequency gain margin such that the system reached a persistent LCO. The AAC was expected to decrease $K_{T}$ such that the system regains stability $\left(K_{T}<1\right)$.

\section{A. Experimental Results}

For each run, the test operator held the IP close to its unstable equilibrium condition before starting the experiment. Figure 11 shows the results for the "do no harm" case in which the "gain shift" parameter was set to 1 . The IP angle and cart position hovered about their equilibrium points respectively and the AAC-modulated $K_{T}$ stayed around unity as expected, minimal adaptation. 

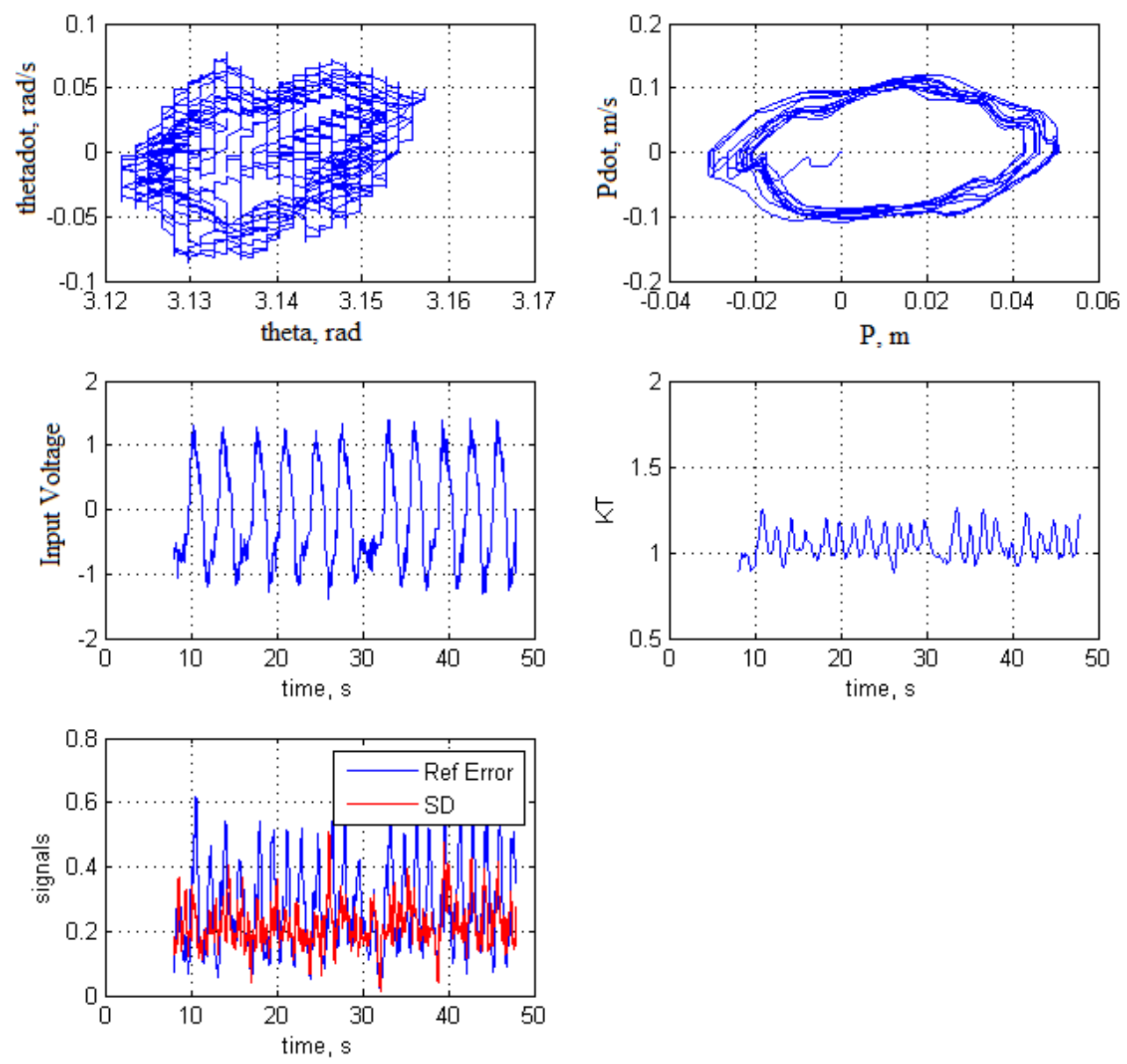

Figure 11. Hardware Case 1: "gain shift" parameter $=1(0 \mathrm{~dB})$

Figure 12 shows results for the reference tracking case in which the "gain shift" parameter was reduced by a factor of 0.4 , or roughly $-8 \mathrm{~dB}$. Without the AAC active, system operated with a negative low frequency gain margin and the nominal controller was unable to keep the IP upright. With the AAC on, the algorithm immediately sensed a difference between the reference model and the gyro output and increased $K_{T}$ to an average value of 1.7 and kept the system stable. Subplot 12.3.2 shows the reference model error dominating the spectral damper error, which led to the gain up behavior.

The adaptation limit of the $\mathrm{AAC}$ is based on the $\pm 6 \mathrm{~dB}(\times 0.5$ to $\times 2)$ gain margin requirements specified for the baseline controller. In one of the experiments, the "gain shift" parameter was dropped to 0.27 to demonstrate the behavior of the AAC at its upper limit of $\times 2$. As expected, the AAC-modulated $K_{T}$ stayed around 2 and the system remained stable. These results are shown in Figure 13. A further drop in the "gain shift" parameter would exceed the capabilities of the AAC. In other words, the AAC algorithm is in essence "extenting" the gain margins of the baseline linear controller as discussed in Ref. 9. 

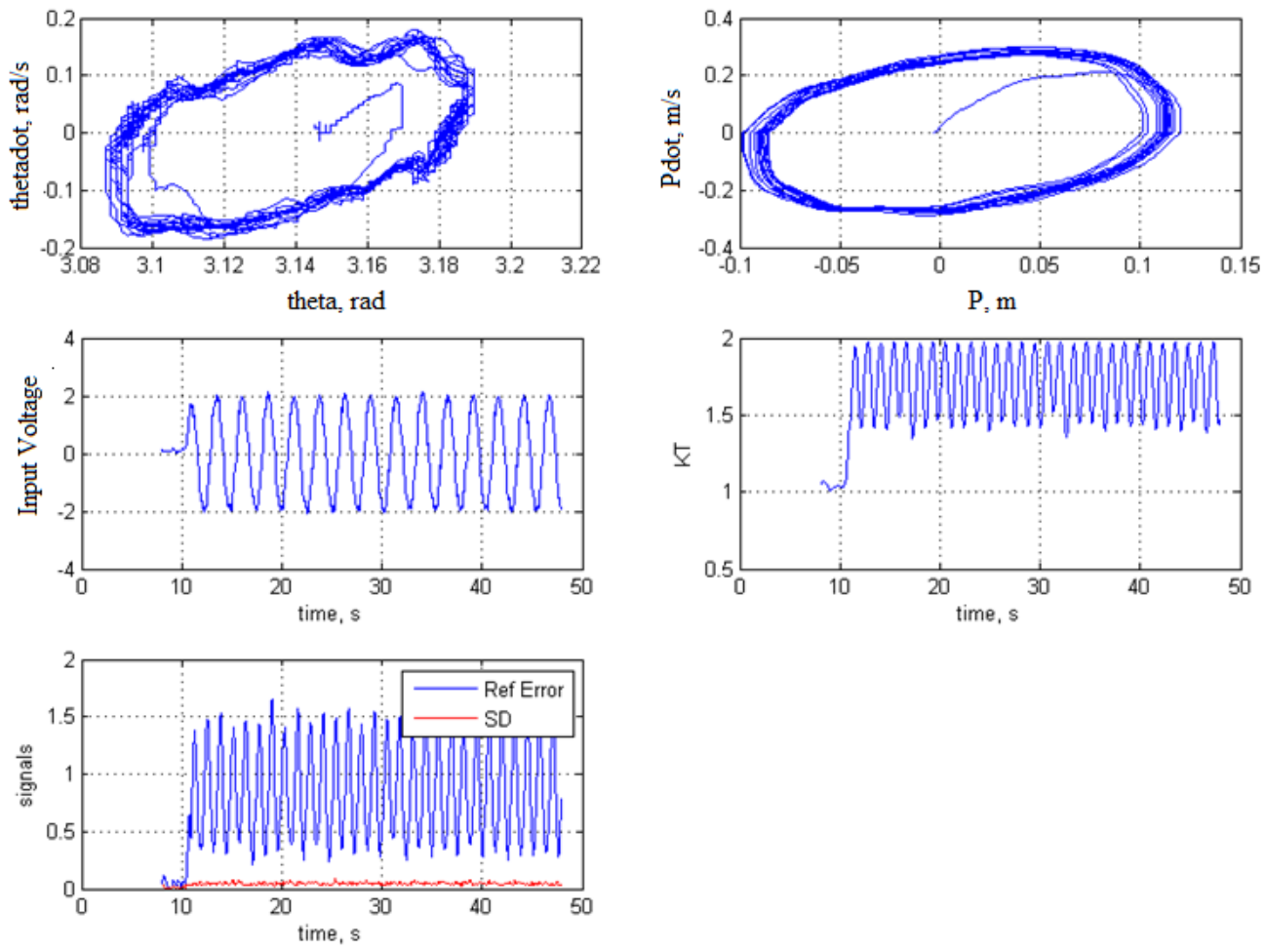

Figure 12. Hardware Case 2a: "gain shift" parameter $=0.4(-8 \mathrm{~dB})$
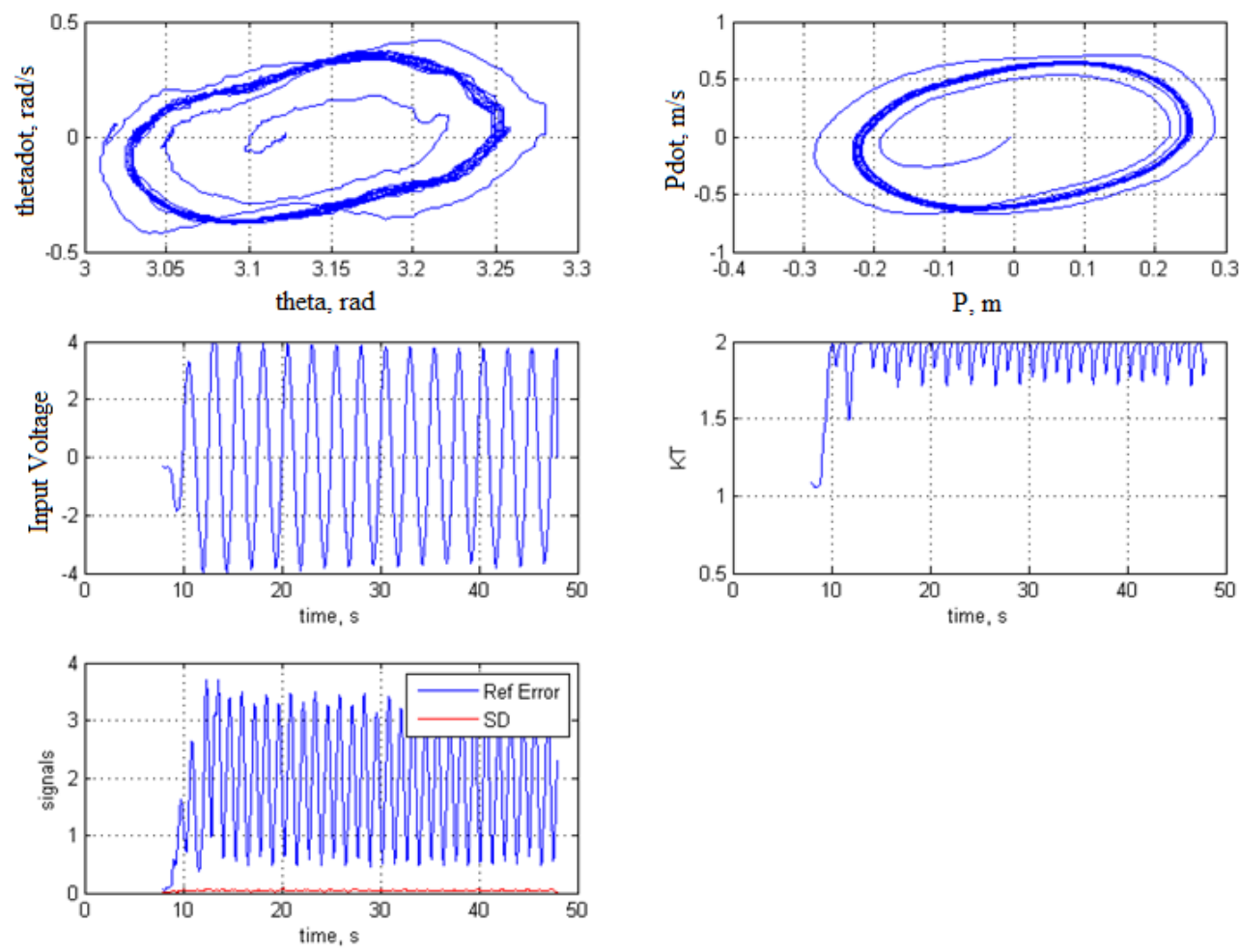

Figure 13. Hardware Case 2b: "gain shift" parameter $=0.27(-11.37 \mathrm{~dB})$ 
Figure 14 shows results for the undesirable parasitic dynamics case in which the "gain shift" parameter was increased by a factor of 4.2 such that the high frequency gain margin was violated. Without the AAC active, the system operated with a $0 \mathrm{~dB}$ high frequency gain margin, the control input saturated, and the IP sustained a $7 \mathrm{~Hz}$ limit cycle oscillation as shown in Fig. 9. With the AAC on, $K_{T}$ was reduced to an average value of 0.8 , and the control input was no longer saturated. In this case, the spectral damper signal dominated the reference model error signal, which led to the expected gain down behavior.

Similar to case 2, experiments were performed to assess the behavior of the AAC near its lower limit $(\times 0.5)$. In theory, the "gain Shift" parameter can be increased by a factor of $8.4(18.5 \mathrm{~dB})$ which out cause the AAC-moduldated $K_{T}$ to bottom out at 0.5 . In practice, $K_{T}$ bottomed out with a "gain Shift" parameter of $\times 7.5$ as the input reached the saturation limit shown in Figure 15. This is minor discrepancy is likely caused by model uncertainties.
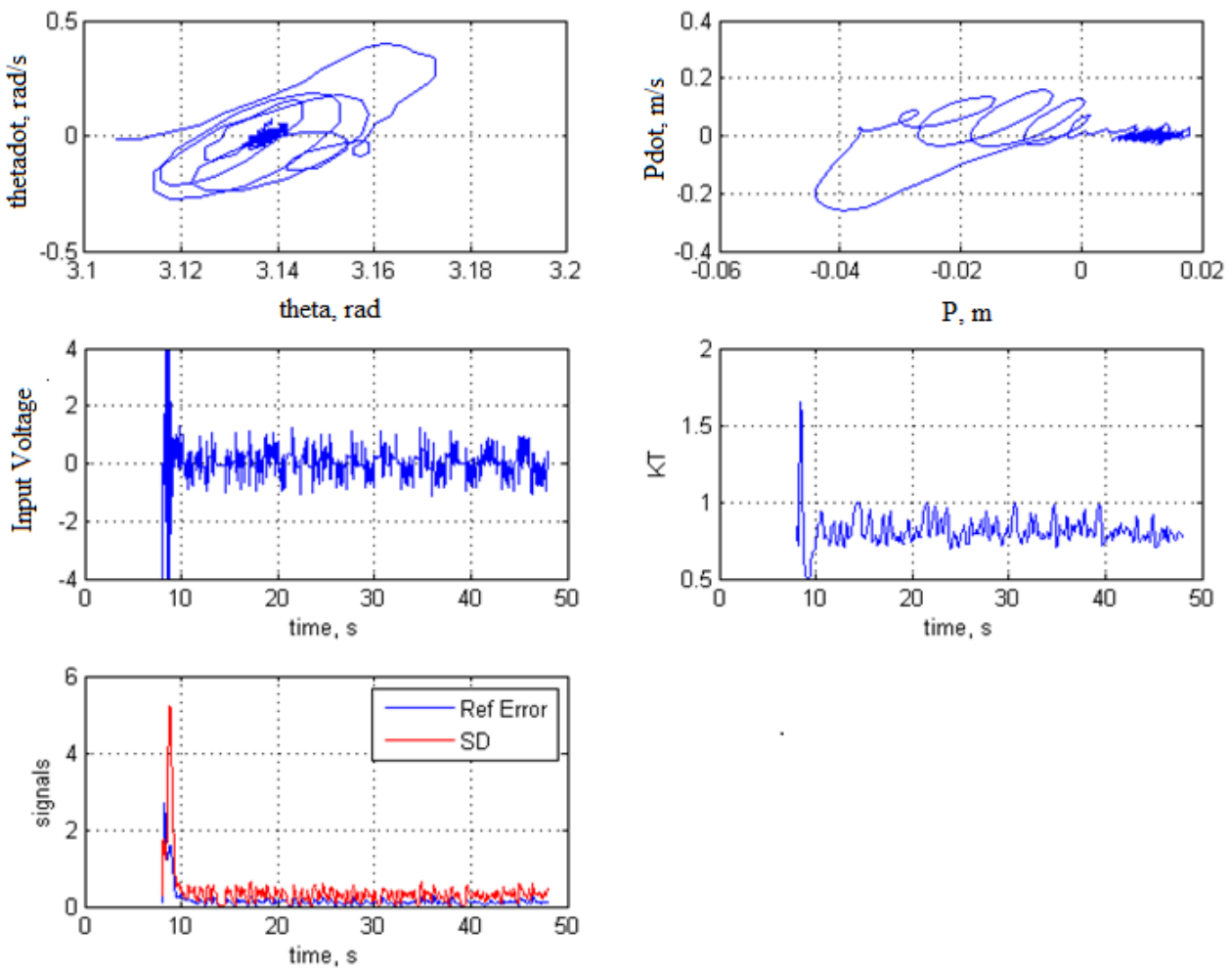

Figure 14. Hardware Case 3a: "gain shift" parameter $=4.2(12.5 \mathrm{~dB})$ 

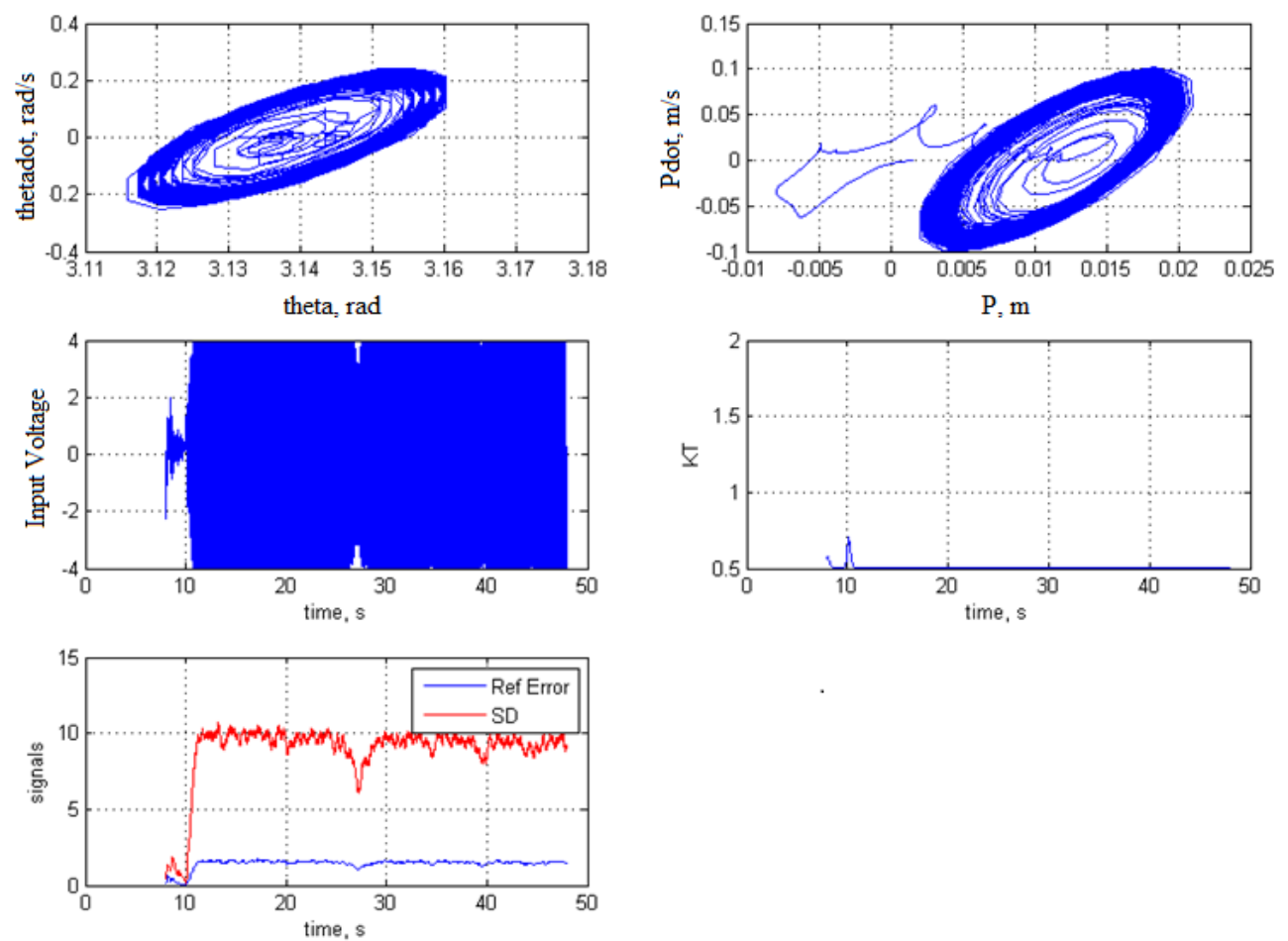

Figure 15. Hardware Case 3b: "gain shift" parameter $=7.5(17.5 \mathrm{~dB})$

As described in Ref. 9, there are cases involving excessively high or low forward loop gain at which the $\mathrm{AAC}$ is required to continuously operate at the extreme boundaries (in the event of a persistent disturbance) can result in continually modulation of $K_{T}$ as the adaptive algorithm seeks to maintain closed loop stability. This magnifest itself in a gain oscillation phenomenon and is at times referred to as "gain swell" and appears to be benign through extensive simulation studies. Figure 16 shows the "gain swell" phenomenon on the pole-cart setup when the "gain shift" parameter was increased by a factor of 7.2. Majority of the time, the AAC-modulated $K_{T}$ stayed close to the lower adaptaion limit of 0.5 , however there were time it exhibited sporadical spikes. Subsequently this caused the control input to go in and out of saturation. This phenomenon is repeatable and consistent with what was observed in the simulation results performed by the SLS program. 

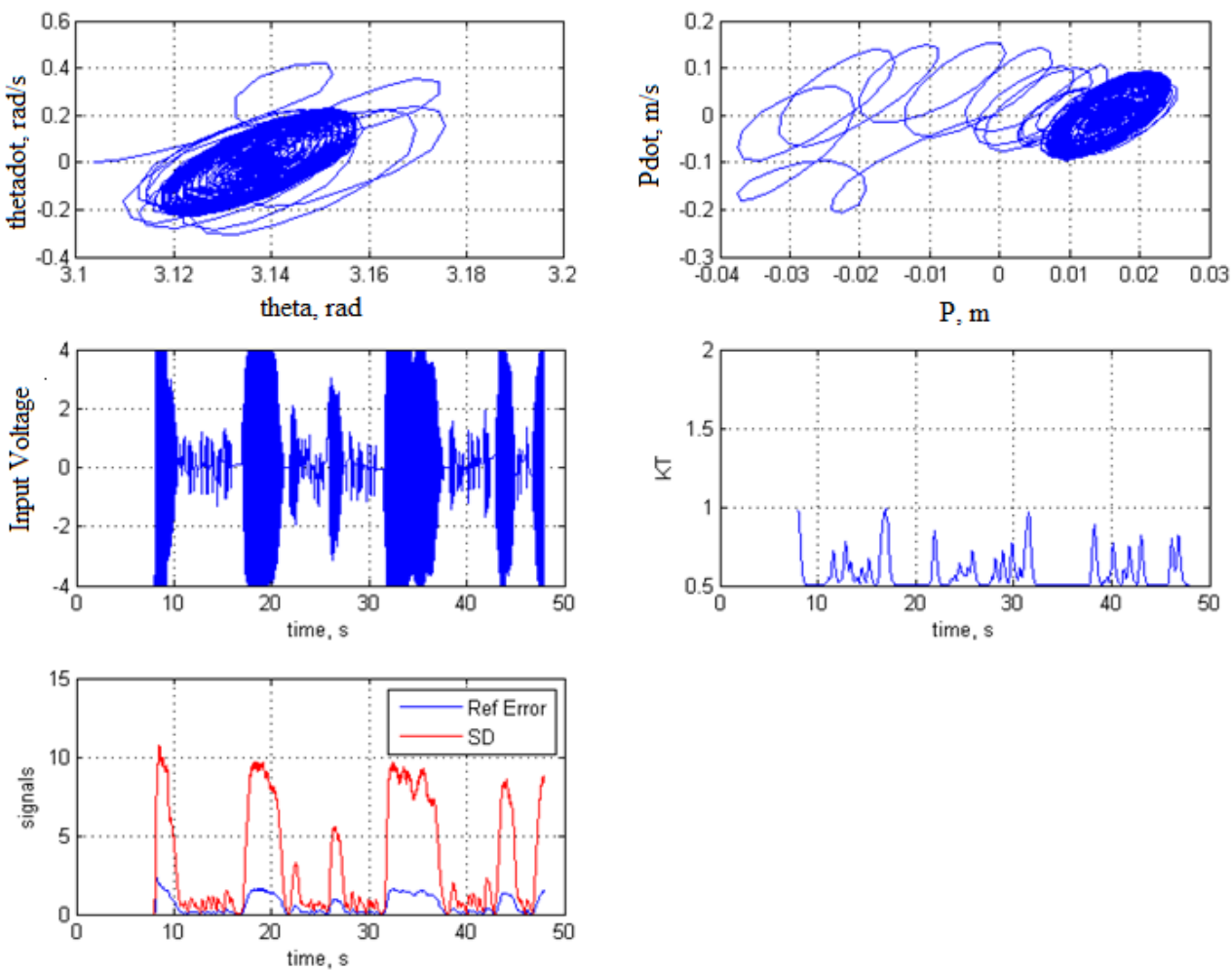

Figure 16. Hardware Case 3c: "gain shift" parameter $=4.2(17.14 \mathrm{~dB})$

\section{B. Numerical Results}

Numerical simulation for the same test cases described in the Experimental Results subsection were performed. Selective results are shown in this section. In general, the simulation results matched fairly well with experiment. Figure 17 shows simulation results for the "do no harm" case at which the "gain shift" parameter was set to 1 . In the absence of any external disturbances or unmodeled dynamics, the IP angle and cart position immediately converged to their respective equilibrium locations, and the AAC-modulated $K_{T}$ stayed at unity as expected. Whereas with the experiment, the AAC modulated $K_{T}$ oscillated slightly about 1 as shown in Fig. 11. The initial spike in the adaptive gain is due to an initial condition that was away from the equilibrium point. 

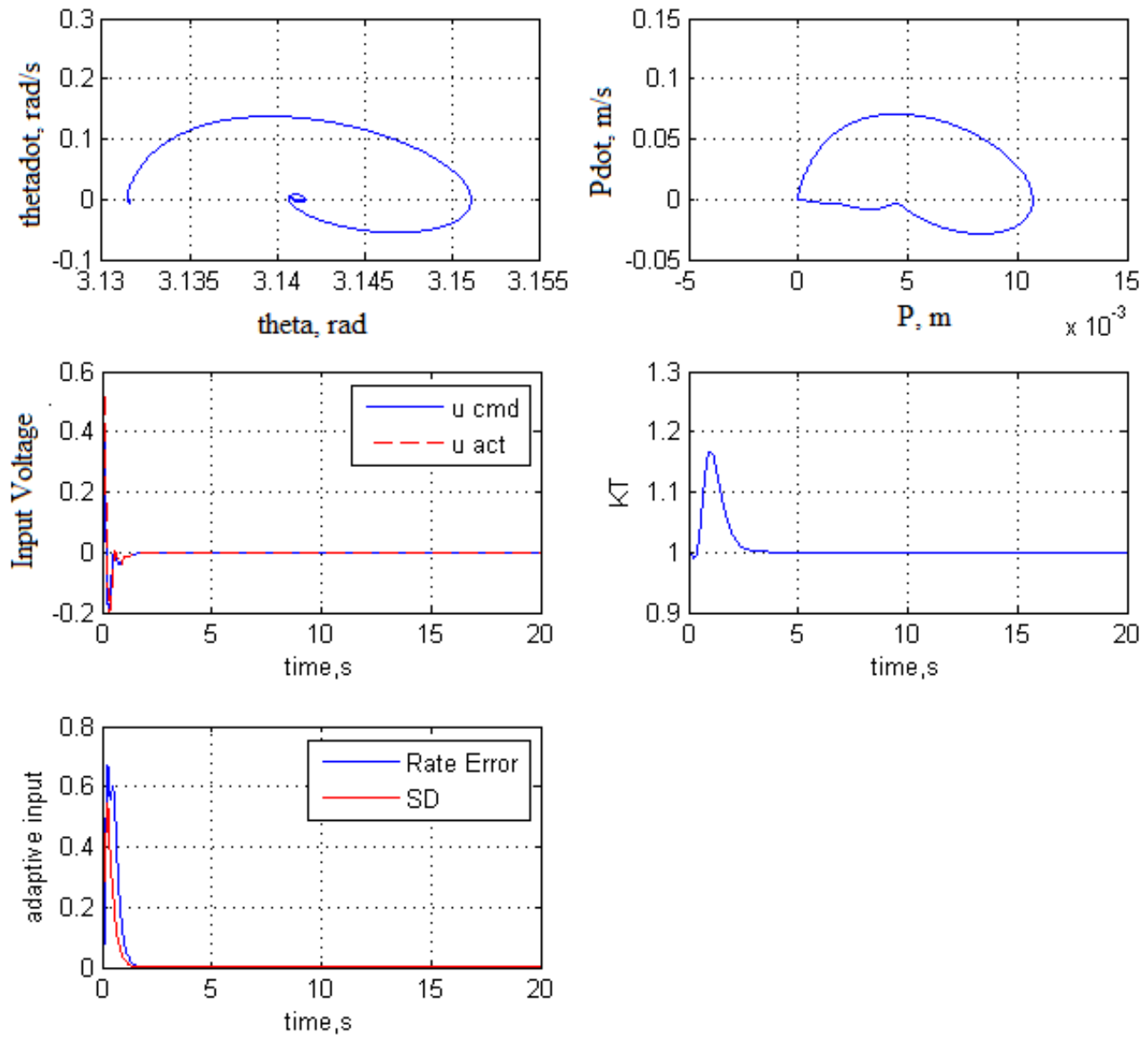

Figure 17. Simulation Case 1: "gain shift" parameter $=1(0 \mathrm{~dB})$

Figure 18 shows the simulation results for case $2 \mathrm{~b}$ with the "gain shift" parameter set to 0.23 . This was intended to assess the behavior of the system as the AAC operates close to its gain up limit of $\times 2$. The simulation results compare well with experimental results shown in Fig 13 with the reference model error dominating the spectral damper error causing an expected gain up behavior. The "gain swell" phenomenon described in the previous section can also be seen in the $K_{T}$ time history. Figure 19 shows results for case 3 in which the "gain Shift" parameter was set to 9. Here, the AAC gain oscillates close to its lower limit of 0.5 and is able to keep the control input from saturation. 

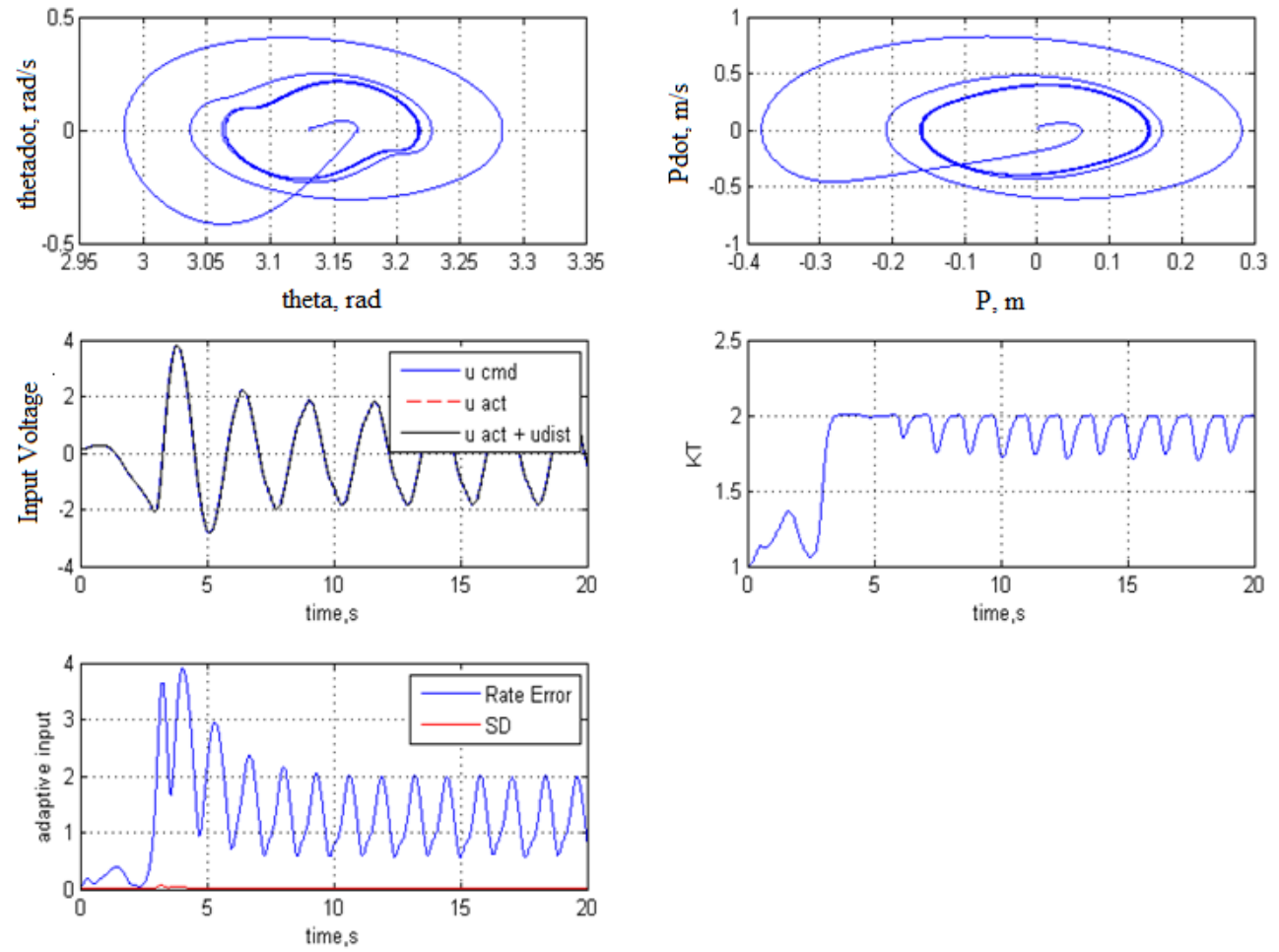

Figure 18. Simulation Case 2: "gain shift" parameter $=0.23(-12.76 \mathrm{~dB})$
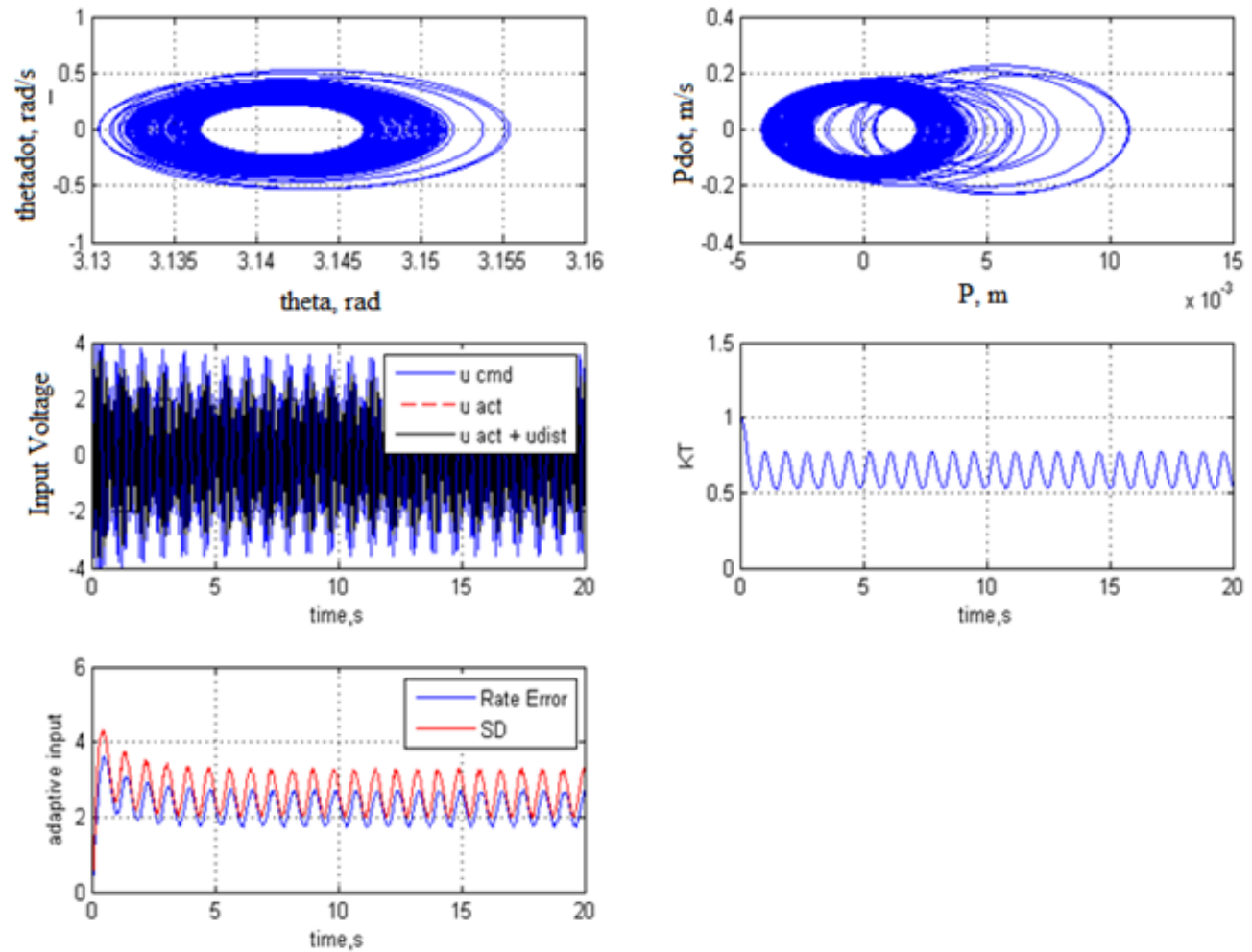

Figure 19. Simulation Case 3: "gain shift" parameter $=9(19.08 \mathrm{~dB})$ 


\section{Conclusions}

The SLS adaptive augmenting controller (AAC) was designed to provide an extra layer of robustness to the baseline fixed-gain controller by "extending" the classical stability margins. The dynamical similarities between the pole-cart system with an aerodynamically unstable rocket makes it an excellent test platform for the AAC algorithm. This study was part of a SLS-NESC joint risk-reduction effort to further gain confidence into the AAC design, operation, robustness, and application. Thus far, all three key features of the AAC algorithm were successfully demonstrated on the pole-cart hardware and numerical simulation. The AAC modulates the forward loop gain, $K_{T}$, depending on a balance of model reference tracking error with the spectral damper component, level of high frequency content in the control input signal. In the "do no harm" case, the baseline controller was performing nominally and minimal adaptation from AAC was required. In the reference model tracking case, the "gain shift" parameter was intentionally reduced such that the nominal controller was no longer capable of stabilizing the IP. The adaptive controller sensed the large reference model error in real-time and increased the forward loop gain, $K_{T}$ such that the system regained stability. In the parasitic dynamics case, the "gain shift" parameter was intentionally increased such that the nominal high frequency gain margin was violated and system entered a persistent limit cycle oscillation (LCO). The AAC sensed the high frequency content in the control signal and reduced $K_{T}$ in real-time to cause the system to exit the LCO. Overall, the AAC behaved exactly as intended for the three primary design objectives.

\section{References}

${ }^{1}$ Orr, J. and VanZwieten, T., "Robust, Practical Adaptive Control for Launch Vehicles," No. 2012-4549, AIAA, 2012.

${ }^{2}$ J. Wall, J. O. and VanZwieten, T., "Space Launch System Implementation of Adaptive Augmenting Control," No. AAS 14-051, AAS, 2014.

${ }^{3}$ T. VanZwieten, E. Gilligan, J. W. J. O. C. M. and Hanson, C., "Adaptive Augmenting Control Flight Characterization Experiment on an F/A-18," No. AAS 14-052, AAS, 2014.

${ }^{4}$ J. Wall, T. VanZwieten, E. G. C. M. C. H. and Orr, J., "In-Flight Suppression of a Destabilized F/A-18 Structural Mode Using the Space Launch System Adaptive Augmenting Control System," No. 2015-1775, AIAA, 2015.

${ }^{5}$ Buonocunto, P. and Corucci, F., "Real-Time PID control of an Inverted Pendulum," MS Thesis, University of Pisa, Italy.

${ }^{6}$ Krishnan, R., "On Stabilization of Cart-Inverted Pendulum System, An Experimental Study," MS Thesis, National Institute of Technology, Rourkela, India.

${ }^{7}$ Tedrake, R., "Underactuated Robotics Class Notes," MIT.

${ }^{8}$ Spong, M., "Underactuated Mechanical System," Tech. rep., University of Illinois of Urbana Champaign, 1997.

9 "Space Launch System Program Integrated Guidance, Navigation, and Control Performance Assessment Volume 4," Tech. rep., NASA, March 2015.

${ }^{10}$ T. VanZwieten, M. H. and Wall, J., "Evaluating the Stability of NASA's Space Launch System with Adaptive Augmenting Control," ESA GNC, 2017.

${ }^{11}$ Shaub and Junkins, Analytical Mechanics of Space Systems, AIAA, 2003. 